\title{
Modeling Reaction and Diffusion Processes of Fuel Cells within Modelica
}

\author{
Kevin L. Davies Comas L. Haynes Christiaan J.J. Paredis \\ Georgia Institute of Technology, Woodruff School of Mechanical Engineering \\ Atlanta, Georgia USA
}

\begin{abstract}
The field of fuel cell (FC) technology offers a challenging and rewarding application for the Modelica language because it is highly multi-disciplinary and it entails physical phenomena (e.g., catalysis) that are not fully understood. Modelica is a valuable platform from which to explore FCs because it is appropriate for the representation of physical interactions. This paper describes elements of a FC library which has been developed in Modelica. The goal of the modeling effort is to take full advantage of the physically representative nature of the Modelica language. To this end, it is important for the models to be consistent and explicit in terms of energy and species balances. The paper emphasizes the representation of diffusion and electrochemical processes. In these areas, the traditional approach is to represent empirically observed behavior, and this is not necessarily rigorous from the standpoint of energy and species balances. To describe the diffusion and electrochemical processes in a form that is suitable for Modelica, alternative and possibly more physically fundamental model equations have been developed. Keywords: media; streams; diffusion; fuel cell
\end{abstract}

\section{Introduction}

Traditionally, FC models have been developed with the goal of capturing behavior that has been observed empirically [13]. However, a model that is empirically representative under a certain range of conditions may not be representative under others. Moreover, empirically-based models are not necessarily consistent in terms of energy and species balances.

The goal of the research described in this paper is to create a robust, flexible, and dynamic model of FCs in the Modelica language. In order to take full advantage of the Modelica language, it is important for the model to be consistent and explicit in terms of energy and species balances. This presents both a challenge and an opportunity. Problems may occur if empirically derived equations have inconsistencies and are implemented in Modelica. When these problems are solved, however, a deeper understanding of the fundamental phenomenon may be uncovered.

This paper describes elements of a FC library in Modelica. It emphasizes two areas that have not been fully explored in Modelica: the transport of chemical species by diffusion and electrochemical reactions. Most FC models in the literature describe diffusion via the dusty-gas model or derivatives of it, which are empirically based and may lead to singularities [14]. Also, most FC models describe the anode and cathode jointly, with only the net reaction and a single electric potential. However, this approach is not suitable for the FC library because the anode and cathode catalyst layers are separate and are connected to opposite sides of the proton exchange membrane (PEM).

\section{Model Equations}

\subsection{Media Library}

The Media library defines the relationship between the properties of chemical mixtures. The medias within the library are based on the ideal gas assumption. This assumption is appropriate because FCs typically operate at low pressures and high temperatures with respect to the critical pressures and temperatures of the gases that are present, with the exception of $\mathrm{H}_{2} \mathrm{O}$. Depending on a model option, $\mathrm{H}_{2} \mathrm{O}$ is either described as an equilibrium mixture of an incompressible liquid and an ideal gas or as a non-condensing ideal gas. A media is specified within every interface and 
higher-level model of the FC library. In the anode, the media represents a mixture of $\mathrm{H}_{2} \mathrm{O}$, hydrogen $\left(\mathrm{H}_{2}\right)$, and carbon monoxide $(\mathrm{CO})$. In the cathode, it represents $\mathrm{H}_{2} \mathrm{O}$, oxygen $\left(\mathrm{O}_{2}\right)$, and nitrogen $\left(N_{2}\right)$. At each instantiation, a minimal set of media properties is defined by the conditions within the interface or element. This set, the state, is sufficient to uniquely determine all other intensive properties of the fluid. As Gibb's phase rule [7] states, $N_{F}=2+N_{S}-N_{P}$, where $N_{F}$ is the number of degrees of freedom, $N_{S}$ is the number of species, and $N_{P}$ is the number of phases present. For example, the cathode fluid contains $\mathrm{H}_{2} \mathrm{O}, \mathrm{O}_{2}$, and $N_{2}$, which means that $N_{F}=3$. Therefore, $N_{D}=3$ if liquid and gas are both present, but $N_{F}=4$ if the fluid is entirely gaseous.

The varying degrees of freedom require careful attention. The Modelica language currently does not support models with variable structure [4]. In addition, the media and the element with which it interacts are separate classes within the object-oriented model structure, and the interface between the two should be the same regardless of the number of phases that are present in the fluid. Since Gibb's phase rule only applies to the intensive properties of the fluid, $N_{P}-1$ additional equations must be given to specify both the intensive and extensive properties of the fluid. For example, even though either pressure or temperature is sufficient to specify the intensive properties of saturated $\mathrm{H}_{2} \mathrm{O}$ (i.e., on or within the two-phase boundary), an additional property such as water quality (the fraction of $\mathrm{H}_{2} \mathrm{O}$ that is vaporized) must be given in order to specify the extensive volume of the $\mathrm{H}_{2} \mathrm{O}$.

The following equations allow the simulation of both one-phase and two-phase regions. Within the mixture, the molar concentration of $\mathrm{H}_{2} \mathrm{O}$ is the sum of the molar concentrations of liquid $\mathrm{H}_{2} \mathrm{O}$ and $\mathrm{H}_{2} \mathrm{O}$ vapor: $\mathrm{C}_{\mathrm{H}_{2} \mathrm{O}}=\mathrm{C}_{\mathrm{H}_{2} \mathrm{O}(l)}+$ $\mathrm{C}_{\mathrm{H}_{2} \mathrm{O}(\mathrm{g})}$. Water is assumed to be the only condensing chemical species within the mixture, so the fraction of $\mathrm{H}_{2} \mathrm{O}$ within the gas phase is $C_{g\left(H_{2} \mathrm{O}\right)}=C_{H_{2} O(g)} /\left(1-C_{H_{2} O(l)}\right)$. Water quality is expressed as $x=C_{H_{2} O(g)} / C_{H_{2} O}$. In the liquid/gas region, the $\mathrm{H}_{2} \mathrm{O}$ is saturated, that is, $C_{H_{2} O(g)} p=p_{l \mid g}(T)$, where $p_{l \mid g}(T)$ is the saturation pressure as a function of temperature, defined in the Modelica Standard Library (Modelica library) [6] as $p_{l \mid g}(T)=611.657 \exp (17.2799-$ $4102.99 /(T-35.719))$. From the preceding equa- tions, $C_{H_{2} O(g)}=1-\left(p C_{H_{2} O(g)}\right) / p_{l \mid g}(T)$ in the liquid/gas region.

If $C_{\mathrm{H}_{2} \mathrm{O}} p>p_{l \mid g}(T)$, then liquid is present. If $p>$ $p_{l \mid g}(T)$ and $C_{H_{2} O}=1$ (i.e., the media is entirely $\mathrm{H}_{2} \mathrm{O}$, without any non-condensing gases present), then the mixture is entirely liquid. The behavior of the phases of the fluid can be summarized as:

$$
C_{H_{2} O(l)}=\left\{\begin{array}{l}
C_{H_{2} O} \\
\quad \text { if } C_{H_{2} O} p>p_{l \mid g}(T) \text { and } C_{H_{2} O}=1 \\
1-p C_{H_{2} O(g)} / p_{l \mid g}(T) \\
\quad \text { if } C_{H_{2} O} p>p_{l \mid g}(T) \text { and } C_{H_{2} O}<1 \\
0, \quad \\
\quad \text { if } C_{H_{2} O} p \leq p_{l \mid g}(T)
\end{array}\right.
$$

The state variables of the media are typically chosen to be the amounts of the chemical species present within a control volume (e.g., expressed in total moles and the molar concentrations of all but one chemical species in the mixture) and temperature. These variables are sufficient to specify all the intensive and extensive properties of the fluid, regardless of whether the fluid is in the one-phase or two-phase region. The continuity of these variables across the discrete events generated by the Boolean conditions in Eq. 1 helps the simulation solver run more efficiently.

The enthalpy and entropy of the chemical species in their pure forms at standard pressure are calculated based on the models in the Modelica library, which implement the equations and empirical data presented by McBride et al. [3]. Dynamic viscosity is also calculated based on the models in the Modelica library. Several other properties including relative humidity $(\mathrm{RH})$, molar mass, Gibbs free energy of chemical species in their pure form at reference pressure, and molar volume or specific volume of the phases and entire mixture are determined from thermodynamics [7]. The properties of the entire mixture are determined by a weighted average of the intensive properties of each chemical species [7].

\section{$2.2 \quad$ Interfaces}

Acausal interfaces describe fluid, thermal, and electronic interactions within the FC. Each interface has an equal number of flow (through) and property or potential (across) variables, but no equations. 
FluidPort The FluidPort interface describes the flow of chemical mixtures. The flow variables represent chemical species and enthalpy. There are two versions of FluidPort interface. The first version is that of the Modelica_Fluid library [5], which uses a mass basis in order to be compatible with other components such as pumps and valves. The second version, which has been developed for the FC library, uses a mole basis so that processes such as diffusion and chemical reactions may be described directly. The property variables are pressure, specific enthalpy, and the molar concentrations of all but one of the chemical species. Other intensive properties of the mixture passing through the interface are specified by the Media library described above in Section 2.1, and the extensive properties are specified by the model of the control volume, described below in Section 2.3.1.

HydrationPort The HydrationPort interface is similar to the mole based version of the FluidPort interface, but is specific to the flow of $\mathrm{H}_{2} \mathrm{O}$ as an absorbed medium through a bulk material (e.g., the PEM). The HydrationPort interface has been developed for the FC library. The flow variables are the molar flow rate of $\mathrm{H}_{2} \mathrm{O}$ and the enthalpy flow rate. The property variables are temperature and hydration. In the case of the PEM, hydration is expressed as the ratio of moles of $\mathrm{H}_{2} \mathrm{O}$ to moles of sulfonic acid $\left(\mathrm{SO}_{3}^{-}\right)$groups.

HeatPort The HeatPort interface describes heat flow; the potential variable is temperature. The HeatPort interface and the following Pin interface are instantiated directly from the Modelica library.

Pin The Pin interface describes electronic flow or protonic flow, and the potential variable is electric potential.

\subsection{Elemental Models}

All of the elemental models include the applicable terms of the energy balance and species balance equations. In the following equations, $i$ is the index of the fluid interfaces, $j$ is the index of the thermal interfaces, and $k$ is the index of the chemical species.

$$
\frac{\mathrm{d} u}{\mathrm{~d} t}=i \Delta \phi+\sum_{i} \dot{h}_{\rightarrow i}+\sum_{j} \dot{u}_{\rightarrow j}
$$

$$
\frac{\mathrm{d} n_{k}}{\mathrm{~d} t}=\dot{n}_{k, r e a c t}+\sum_{i} \dot{n}_{i, k}
$$

The storage models (Section 2.3.1) are dynamic, and include the storage term on the left hand side of Eqs. 2 and 3. The process models (Sections 2.3.2 and 2.3.3) are static and do not include the storage term. The enthalpy flow rate through interface $i$ is specified as a sum of the enthalpy of the chemical species:

$$
\dot{h}_{k}=\sum_{k} \bar{h}_{i, k} \dot{n}_{i, k}
$$

\subsubsection{Storage Models}

The storage models integrate the energy and species, flowing into or out of a control volume over time in order to determine the present state, according to Eqs. 2 and 3. Each storage model has at least one time-varying state variable, which describes the condition of the element at a given time.

PoreVolume The PoreVolume model describes the storage of internal energy and chemical species. The properties are determined by the selected media. The time-varying state variables depend on the usage scenario, but are typically temperature, total moles, and the volumetric concentrations of all but one of the chemical species.

MembraneVolume The MembraneVolume model describes the storage of internal energy and $\mathrm{H}_{2} \mathrm{O}$ in the PEM. The states are temperature and total moles of $\mathrm{H}_{2} \mathrm{O}$. The hydration of the PEM $(\lambda)$ is related to $n_{\mathrm{H}_{2} \mathrm{O}}$, the amount of $\mathrm{H}_{2} \mathrm{O}$ in the control volume, by the following equation.

$$
\lambda=n_{H_{2} O} \mathbf{M}_{d r y P E M} /\left(\rho_{d r y P E M} A \mathrm{~d} L_{\cdot z}\right)
$$

PortVolume The PortVolume model is instantiated directly from the Modelica_Fluid library. It is similar to a PoreVolume model, but it accounts for the storage of fluid on a mass basis rather than a mole basis.

Capacitor and HeatCapacitor The Capacitor and HeatCapacitor models are instantiated directly from Modelica library to describe electrical and thermal storage, respectively. 


\subsubsection{Reaction Models}

The reactions of the anode and cathode are modeled separately. The rates of energy (in electrical, chemical, and thermal forms) are balanced by Eq. 2. The flow rates of the species (in electronic, protonic, and chemical forms) are balanced by the stoichiometric ratio of the reactions and Eq. 3. The reaction models take direct advantage of the media properties from McBride et al. [3], which are available in the Modelica library.

The reaction models use a new, and possibly more fundamental, form of an electrochemical rate equation which can be contrasted with the widelyused Butler-Volmer equation. The new form is based on the hypothesis that the need for a symmetry factor in the Butler-Volmer equation is actually an artifact of the assumption of equal and opposite biases in the forward and backward directions. The traditional approach is to calculate the Nernst potential and add an "activation overpotential". The Nernst potential is dependent on temperature but not pressure or current, because it assumes standard pressure $\left(p=p^{0}\right)$ and an open-circuit condition $(i=0)$. The overpotential accounts for the actual pressure and current. It is typically negative because it decreases with increasing current, decreasing partial pressure of the reactants, and increasing partial pressure of the products. The new electrochemical rate equation is simpler to implement in the Modelica language than a Nernst potential/overpotential approach because it does not separate factors that are actually coupled in reality.

There are two traditional methods of calculating the activation overpotential. The first method, which is seemingly the simplest, is the Tafel equation. It combines the forward and backward currents of the reaction by assuming that the forward current dominates under typical operating conditions. This results in a singularity (a natural logarithm of zero at zero net current), which is unfortunate because the open-circuit condition $(i(t=0)=0)$ is a typical and convenient initial condition (IC). The Tafel equation is not acceptable for an acausal reaction model, which certainly must be able to simulate at open-circuit conditions. In reality, a FC and its electronic load are separate components, and the FC is not a singularity before the load is connected to it, when $i=0$.

The Butler-Volmer equation for the activation overpotential handles the open-circuit condition by accounting for the fact that while the net current is zero under the open-circuit condition, there are equal and opposite, but nonzero, diffusion currents in the forward and backward directions. However, the Butler-Volmer equation makes the default assumption that the forward and backward currents are driven by opposite signs of the same bias. That bias is a combination of the Gibbs free energy of the reactants and products, as well as electronic and protonic energies. An "electrode symmetry factor" is then included to account for the fact that the forward and backward bias may not actually be symmetric. The symmetry factor is typically found to be approximately 0.5 for the net reaction. However, in the present FC model, the anode and cathode reactions must be modeled separately since the anode and cathode catalyst layers are separate models. During the development of the model, it was found that the anode symmetry factor of 0.5 produces an unrealistic anode electric potential, which results in an incorrect cell electric potential when the anode and cathode are electrically connected in series to represent the FC.

Therefore, the Butler-Volmer equation is modified under the premise that the power of one of the exponential terms in the Butler-Volmer equation should be associated only with the energies of the reactants needed for the forward direction of the reaction. Accordingly, the power of the second exponential term should be associated with the energies of the reactants for the backward direction of the reaction, i.e., the products of the forward direction of the reaction. It is also realized that the electric potential of the reaction is related to the sum of an electronic energy and a protonic energy. The electrons $\left(e^{-}\right)$and protons $\left(\mathrm{H}^{+}\right)$are either reactants or products, depending on the reference direction of the reaction.

The exponential factors of the electrochemical rate equation are of the form $\exp (g / \mathbf{R} T)$, but are typically written in the form $\left(p / p^{0}\right) \exp \left(g^{0} / \mathbf{R} T\right)$, which is equivalent under the assumption of an ideal gas. This equivalence can be shown by realizing that Gibbs free energy can be defined as $g=h-T s$. Enthalpy is defined via $\mathrm{d} h=T \mathrm{~d} s+$ $\mathrm{v} \mathrm{d} p$, which can be reduced under the assumption of an ideal gas and integrated under isothermal conditions to give $s-s^{0}=-\mathbf{R} \ln \left(p / p^{0}\right)$, where $s^{0}$ is the entropy at standard pressure $\left(p^{0}\right)$. 
Gibbs free energy can therefore be represented as a value at standard pressure $\left(g^{0}=h-T s^{0}\right)$ and an offset, such that $g=g^{0}-T\left(s-s^{0}\right)$, or $g=g^{0}+\mathbf{R} T \ln \left(p / p^{0}\right)$. Therefore, $\exp (g / \mathbf{R} T)=$ $\left(p / p^{0}\right) \exp \left(g^{0} / \mathbf{R} T\right)$ if the media behaves as an ideal gas.

When the reaction models are formulated under these premises, it is no longer necessary to calculate a Nernst potential at all. The new electrochemical rate equation results in the electrode electric potential directly. Also, the need for two model parameters (the symmetry factors of the anode and cathode) is eliminated. This offers the advantage that fewer parameters must be specified to utilize the FC model. The new electrochemical rate equations are more robust than the Tafel equation because the natural log of zero cannot occur unless the media properties are miscalculated.

ORR An ORR model describes the oxygen reduction reaction (ORR) whereby oxygen is consumed and $\mathrm{H}_{2} \mathrm{O}$ is produced: $\frac{1}{2} \mathrm{O}_{2}+2 \mathrm{H}^{+}+2 e^{-} \rightarrow \mathrm{H}_{2} \mathrm{O}$. Bockris et al. give an equation [2, Eq. 7.7] for the forward current density of an electrochemical reaction involving $\mathrm{Ag}^{+}$:

$i^{\prime \prime} \rightarrow=\mathbf{F} \frac{\mathbf{k} T}{\mathbf{h}}\left[n^{\prime \prime}{ }_{A g^{+}}\right] \exp \left(-\frac{\Delta \bar{g}^{0}}{\mathbf{R} T}\right) \exp \left(-\frac{\beta \Delta \phi \mathbf{F}}{\mathbf{R} T}\right)$

The equation is adapted here for the ORR and modified to a more fundamental form. It is noted that the concentration factor should strictly include all of the reactants - not only $\mathrm{O}_{2}$ and $\mathrm{H}^{+}$, but also $e^{-}$. The concentration factor is the product of the molar concentrations of the reactants raised to the power of the corresponding stoichiometric ratios and divided by the molar volume of the mixture. In Eq. 6, $n^{\prime \prime} \mathrm{Ag}^{+}$is the surface concentration of $\mathrm{Ag}+$. Here, it is desirable to relate the equation to the molar concentration of the chemical species and volumetric concentration of the mixture. A coefficient, $\gamma_{c a}$, is introduced to relate the effective surface concentration of the reactants on the electrode to the volumetric concentration of the chemical species in the catalyst layer. This coefficient is dependent on the geometry of the electrode (e.g., catalyst surface area) and other factors.

The coefficients of the energy terms in the exponential and the powers of the molar concentrations are the stoichiometric ratios of the reaction, so the coefficient of the electronic term is 2 . The voltage loss is only applied to the reaction $\left(e^{-}\right.$only appears as a reactant, not as a product), so the electrode symmetry factor $(\beta)$ is removed. The factors are reordered and regrouped to be clearer in terms of energy.

$$
\begin{aligned}
i_{c a}^{\prime \prime} \rightarrow= & \mathbf{F} \frac{\mathbf{k} T}{\mathbf{h} \gamma_{c a \overline{\mathrm{v}}}}\left[C_{O_{2}}\right]^{0.5}\left[C_{H^{+}}\right]^{2}\left[C_{e^{-}}\right]^{2} \\
& \cdot \exp \left(\frac{0.5 \bar{g}_{O_{2}}{ }^{0}+2 \bar{g}_{H^{+}}{ }^{0}-2 \mathbf{F} \Delta \phi_{c a}}{\mathbf{R} T}\right)
\end{aligned}
$$

Following the same logic, the backward current is given by the equation below, which is a departure from Eq. 7.11 of Bockris et. al [2].

$$
i^{\prime \prime}{ }_{c a} \leftarrow=\mathbf{F} \frac{\mathbf{k} T}{\mathbf{h} \gamma_{c a} \overline{\mathrm{v}}}\left[C_{H_{2} O}\right] \exp \left(\frac{\bar{g}_{H_{2} O^{0}}}{\mathbf{R} T}\right)
$$

The net current is the forward current minus the backward current. Unlike the strict form of Eq. 7, $C_{H^{+}}$and $C_{e^{-}}$are assumed to be unity. The model assumes that the molar concentration of $\mathrm{H}^{+}$is not limiting because, as noted by Wang et al. [11], the molar concentration of $H^{+}$is high in acidic liquid media. The model also assumes that the molar concentration of $e^{-}$is not limiting; the voltage losses due to electronic resistance are included separately elsewhere.

$$
\begin{aligned}
i^{\prime \prime}{ }_{c a}= & \mathbf{F} \frac{\mathbf{k} T}{\mathbf{h} \gamma_{c a} \overline{\mathbf{v}}} \\
& \cdot\left[\left[C_{O_{2}}\right]^{0.5} \exp \left(\frac{0.5 \bar{g}_{O_{2}}{ }^{0}-2 \mathbf{F} \Delta \phi_{c a}}{\mathbf{R} T}\right)\right. \\
& \left.-\left[C_{\mathrm{H}_{2} \mathrm{O}}\right] \exp \left(\frac{\bar{g}_{\mathrm{H}_{2} \mathrm{O}^{0}}}{\mathbf{R} T}\right)\right]
\end{aligned}
$$

HOR An HOR model describes the hydrogen oxidation reaction (HOR) whereby $H_{2}$ is consumed and $e^{-}$and $H^{+}$are produced: $H_{2} \rightarrow 2 H^{+}+2 e^{-}$. Following a similar derivation to that of the ORR model:

$$
\begin{aligned}
& i^{\prime \prime}{ }_{a n}=\mathbf{F} \frac{\mathbf{k} T}{\mathbf{h} \gamma_{a n} \overline{\mathrm{v}}}\left[\exp \left(\frac{2 \mathbf{F} \Delta \phi_{a n}}{\mathbf{R} T}\right)\right. \\
&\left.-\left[C_{H_{2}}\right] \exp \left(\frac{\bar{g}_{H_{2}}{ }^{0}}{\mathbf{R} T}\right)\right]
\end{aligned}
$$

The HOR and ORR models each have only one parameter. The values of $\gamma_{a n}$ and $\gamma_{c a}$ can be uniquely determined by the cathode and cathode 
electric potentials and the intensive properties of the fluid at a single value of current. Experimentally, it is difficult to distinguish the anode and cathode contributions to the IR-free voltage; however, the anode overpotential is typically much lower [1]. It may not be necessary to determine the exact ratio between $\gamma_{a n}$ and $\gamma_{c a}$ because the goal of the model library is to describe the overall FC electric potential-current relationship rather than the electric potential-current relationships of the anode and cathode separately.

\subsubsection{Transport Models}

The flow process models describe the flow of energy and species through the FC, but not the storage of energy or species.

PressureLoss The model of pressure loss describes advection due to a gradient in total fluid pressure. The fluid is assumed to be uniformly mixed at the molar concentrations $C_{j}$ of the upstream interface. The flow rate of chemical species $i$ through the downstream interface $j, \dot{n}_{i, j}$, is given by:

$$
\dot{n}_{i, j}=C_{j} \dot{n}_{j}
$$

The relationship between the flow rate and pressure difference depends on the fluid properties and the flow regime (e.g., laminar or turbulent), which are either assumed to be constant or determined based on the operating conditions and the fluid properties. The flow rate versus pressure loss equations are given in the WallFriction within the Modelica_Fluid library [5]. The PressureLoss model does not affect the behavior of the FC model significantly because the pressure difference across the flow channels of a FC are often negligible with respect to the absolute pressure. Nevertheless, the model is included to separate the flow channel into discrete storage volumes with varying molar concentrations of the species.

DiffusionSurface The DiffusionSurface model represents the surface at the boundary between advection-dominated flows (e.g., the flow of reactants down the flow channel due to a gradient in pressure) and diffusion-dominated flows (e.g., the flow of reactants through the gas diffusion layer (GDL) due to gradients in molar concentration). The molar concentration of chemical species at the diffusive interface is the average of that across the advective flow path.

TransportPorous The TransportPorous model describes flow through a porous material due to gradients in molar concentration and pressure. The following equation is has been developed to describe the flow of gas from Fick's law [17], Darcy's law, and an analogy to laminar flow through a pipe. The gas phase of the chemical species of interest, $i$, is coupled in a binary manner to the $N_{S}-1$ other chemical species.

$$
\dot{n}_{i(g)}=-D_{i} A \frac{\partial n^{\prime \prime \prime}{ }_{i}}{\partial L_{\cdot}}+\frac{\sum_{\substack{j=1 \\ j \neq i}}^{N_{S}} C_{j} \dot{n}_{j(g) \nabla p} / D_{i, j}}{\sum_{\substack{j=1 \\ j \neq i}}^{N_{S}} C_{j} / D_{i, j}}
$$

Optionally, the advective flow of liquid water can be added in parallel to the flow of gas. The flow of liquid water is described according to Eq. 4 in [18]. If the flow of liquid water is included, then the porosity available for the flow of gas is reduced according to $\varepsilon_{v g}=\varepsilon_{v} \mathrm{v}_{g} /\left(\mathrm{v}_{l}+\mathrm{v}_{g}\right)$.

In the literature within the field of proton exchange membrane fuel cells (PEMFCs) [12], the transport process is often described with a viscous flow equation such as Darcy's law and $N_{S}-1$ repetitions of an equation consisting of momentum, Stefan-Maxwell diffusion, and Knudsen diffusion terms. However, this approach is not rigorous in terms of energy. Weber and Newman [14] note that the typical modeling approach, the dusty-gas model, can lead to a singular matrix. The traditional equations for flow through a porous material have been modified to derive Eq. 12, which is suitable for the energy-based Modelica formalism.

The development of Eq. 12 starts with Darcy's law to describe the advective flow of gases through a porous medium over a nominal or superficial cross-sectional area $\left(A^{0}\right)[15]$ :

$$
\dot{\mathrm{v}}=-\frac{\kappa A^{0}}{\mu} \frac{\partial p}{\partial L_{\cdot z}}
$$

Darcy's law is modified to use kinematic viscosity instead of dynamic viscosity $(\mu=\rho \nu)$ and molar flow rate instead of volumetric flow rate $(\dot{v}=\overline{\mathrm{v}} \dot{n})$. Since density is related to molar mass and molar volume $(\rho=\mathbf{M} / \overline{\mathrm{v}})$, the previous equation can be 
rewritten as:

$$
\dot{n}_{g \nabla p}=-\frac{\kappa A^{0}}{\mathbf{M} \nu_{g}} \frac{\partial p}{\partial L_{\cdot z}}
$$

The equation for laminar flow in a circular pipe is modified in a similar way:

$$
\dot{n}_{\nabla p}=-\frac{\pi d^{4}}{128 \mathbf{M} \nu_{g}} \frac{\partial p}{\partial L_{\cdot z}}
$$

The following equation is obtained by substituting the definition of hydraulic diameter in terms of the effective cross-sectional area and wetted perimeter $\left(d=4 A^{\text {eff }} / P\right)$ and recognizing that the perimeter is the circumference of a circle $(P=$ $2 \pi r)$ :

$$
\dot{n}_{\nabla p}=-\frac{\left(A^{e f f}\right)^{4}}{P^{3} r \mathbf{M} \nu_{g}} \frac{\partial p}{\partial L_{\cdot z}}
$$

Comparing Eqs. 15 and 16, the permeability can be written as $\kappa=\left(A^{e f f}\right)^{4} /\left(P^{3} r A^{0}\right)$. The effective cross-sectional area for flow, $A^{e f f}$, is related to the nominal cross-sectional area by $A^{e f f}=\varepsilon_{A} A^{0}$. The areal porosity, $\varepsilon_{A}$, is related to the volumetric porosity by $\left(\varepsilon_{A}\right)^{1 / 2}=\left(\varepsilon_{v}\right)^{1 / 3}$, assuming that the geometry of the pores is isotropic. This assumption is consistent with the Bruggeman correction for tortuosity which leads to $D_{i, j}$ eff $=\left(\varepsilon_{v}\right)^{3 / 2} D_{i, j}$ (Eqs. 41 and 42 in [12]). Assuming that the crosssection of the pores is circular with radius $r$ and volumetric porosity $\varepsilon_{v g}$ available for gas, then the permeability of the medium to gas is given by $\kappa=r^{2}\left(\varepsilon_{v g}\right)^{8 / 3} / 8$.

The total advective flow can be split into flows of the individual chemical species by accounting for the coupling between the chemical species. The coupling is assumed to be binary, so the development starts with the Stefan-Maxwell equation for binary diffusion [16]. Here, $i$ denotes the chemical species of interest and $j$ denotes the other chemical species.

$$
\nabla C_{i}=\sum_{\substack{j=1 \\ j \neq i}}^{N_{S}} \frac{C_{i} C_{j}}{D_{i, j}}\left(v_{j}-v_{i}\right)
$$

The concentration gradient is set to zero here because the diffusive flow is handled separately. The velocity of the flow is related to the molar flow rate $(v=\dot{n} \overline{\mathrm{v}} / A)$. Making these adjustments and solving for the molar flow rate of chemical species $i$ :

$$
\dot{n}_{i(g) \nabla p}=\frac{\sum_{\substack{j=1 \\ j \neq i}}^{N_{S}} C_{j} \dot{n}_{j(g) \nabla p} / D_{i, j}}{\sum_{\substack{j=1 \\ j \neq i}}^{N_{S}} C_{j} / D_{i, j}}
$$

Only $N_{S}-1$ equations in the form of Eq. 18 are unique; the $N_{S}{ }^{\text {th }}$ equation is redundant and consistent. In summary, the $N_{S}+1$ variables characterizing the advective flow of the TransportPorous model $\left(\dot{n}, \dot{n}_{1}, \ldots, \dot{n}_{N_{S}}\right)$ are described by Eq. 16 (with $\left.\kappa=r^{2}\left(\varepsilon_{v}\right)^{8 / 3} / 8\right), N_{S}-1$ equations in the form of Eq. 18, and an additional equation stating the molar flow rate $\left(\dot{n}=\sum \dot{n}_{j}\right)$.

The diffusive flow of each chemical species is given by Fick's law, stated in terms of volumetric concentration of the chemical species and the molar volume of the mixture:

$$
\dot{n}_{i \nabla C}=-D_{i} A \frac{\partial n^{\prime \prime \prime}{ }_{i}}{\partial L_{\cdot z}}
$$

The advective and diffusive flow rates are added for each chemical species to obtain the net flow rate within the TransportPorous model, Eq. 12.

DiffusionMembrane The DiffusionMembrane model represents the diffusion of $\mathrm{H}_{2} \mathrm{O}$ through the PEM. The rate of diffusive flow is proportional to the hydration gradient, as described by Eq. 20 in [10]. The diffusion coefficient is either held constant or related to PEM hydration according to Eq. 22 in [10] and the software code in developed by Springer [8].

ElectroOsmoticDrag The ElectroOsmoticDrag model describes both electro-osmotic drag and resistance to protonic flow in the PEM. The flow of $\mathrm{H}_{2} \mathrm{O}$ through the PEM and the voltage loss across it are both related to the protonic current. As described by Eq. 18 in [10], electroosmotic drag carries $\mathrm{H}_{2} \mathrm{O}$ through the PEM at a rate that is proportional to protonic current. The coefficient of proportionality depends on PEM hydration. The difference in electric potential is also proportional to protonic flow, as described by Ohm's law. The resistance to protonic flow is either constant, described as an empirical function of protonic current, or related to PEM hydration according to Eq. 25 in [10]. The energy balance 
within ElectroOsmoticDrag model accounts for heating due to resistance.

MembraneSurface The MembraneSurface model relates hydration to humidity, or the activity of $\mathrm{H}_{2} \mathrm{O}$, at the surface of the PEM as Springer et al. determined empirically (Eqs. 16 and 17 in [10]).

HeatingResistor A HeatingResistor model describes heat generation and voltage loss due to a constant resistance.

ThermalConductor and ThermalConvection The ThermalConductor model and ThermalConvection models describe heat flow and are instantiated directly from the Modelica library.

\section{Simulation Results}

Figure 1 compares polarization curves with varying test conditions, specifically anode and cathode pressure, flow plate temperature, cathodic reactant flow rate, and anode and cathode RH. The baseline scenario is shown as the solid curve in each plot. Figure 1a shows that cell electric potential increases with increased operating pressure due to higher reactant volumetric concentration (i.e., higher reactant partial pressure), but that the gain decreases with increasing pressure. Figure $1 \mathrm{~b}$ shows that the cell electric potential decreases as the flow plate temperatures and thus the catalyst layer temperatures are increased, due to the decreased change in Gibbs free energy of the reaction (i.e., decreased Nernst potential). Figure 1c shows that electric potential increases as the cathode reactant flow rate is increased, especially in the high current density region, due to increased $\mathrm{O}_{2}$ molar concentration. Figure 1d demonstrates the net effect of two underlying consequences of varying reactant RH. Higher RH leads to increased hydration of the PEM and lower protonic resistance in the PEM, which causes the slope of the curve to become less negative. However, the increase in RH also decreases the molar concentration of the reactants, so the limiting current density decreases. Qualitatively, the trends shown in Figure 1 are consistent with the experimental test results presented in the literature [9] .

\section{Summary}

Most previous FC models have been created with the primary goal of matching the empirical observations of FC operation. In some cases, this has resulted in model equations that are not rigorous on an energy basis. The research presented within this paper modifies some of the traditional FC model equations to be suitable for the acausal, energy-based representation. Modelica is used as a platform to resolve the differences between empirical representations and first-principle relationships pertaining to fuel cells.

Two areas have been emphasized in this paper: the transport of chemical species by diffusion and electrochemical reactions. These areas are central to fuel cell modeling and have not previously been explored to a full extent in Modelica. Most models in the FC literature describe diffusion via the dusty-gas model or derivatives of it, which are empirically based and may lead to singularities [14]. An alternative has been proposed which is explicit in terms of energy balances. Most FC models also describe the anode and cathode jointly. However, this approach is not suitable for the FC library because the anode and cathode catalyst layers are separate and are connected to opposite sides of the PEM. The proposed alternative addresses this need by modifying the traditional Butler-Volmer equation.

\section{Nomenclature}

\section{Symbols}

$\begin{array}{cl}A & \text { Area }\left(/ \mathrm{m}^{2}\right) \\ C & \text { Molar concentration }\left(/ \mathrm{mol} \mathrm{mol}^{-1}\right) \\ D & \text { Diffusion coefficient }\left(/ \mathrm{m}^{2} \mathrm{~s}^{-1}\right) \\ d & \text { Diameter }(/ \mathrm{m}) \\ \mathbf{F} & \text { Faraday's constant }\left(/ \mathrm{Cmol}^{-1}\right) \\ g & \text { Gibbs free energy }(/ \mathrm{J}) \\ \mathbf{h} & \text { Planck's constant }(/ \mathrm{J}) \\ h & \text { Enthalpy }(/ \mathrm{J}) \\ i & \text { Current }(/ \mathrm{A}) \\ \mathbf{k} & \text { Boltzmann's constant }\left(/ \mathrm{J} \mathrm{K}^{-1}\right) \\ L & \text { Length }(/ \mathrm{m}) \\ \mathbf{M} & \text { Molar mass }\left(/ \mathrm{kg} \mathrm{mol}{ }^{-1}\right) \\ N & \text { Number }(/ 1) . \\ n & \text { Amount }(/ \mathrm{mol}) \\ P & \text { Perimeter }(/ \mathrm{m})\end{array}$

$A$ Area $\left(/ \mathrm{m}^{2}\right)$.

$C$ Molar concentration $\left(/ \mathrm{mol} \mathrm{mol}^{-1}\right)$.

$D$ Diffusion coefficient $\left(/ \mathrm{m}^{2} \mathrm{~s}^{-1}\right)$.

$d$ Diameter $(/ \mathrm{m})$.

F Faraday's constant $\left(/ \mathrm{Cmol}^{-1}\right)$

$(/ \mathrm{J})$

h Planck's constant $(/ \mathrm{J} \mathrm{s})$

Enthalpy $(/ \mathrm{J})$

$i$ Current $(/ \mathrm{A})$.

k Boltzmann's constant $\left(/ \mathrm{J} \mathrm{K}^{-1}\right)$.

$L \quad$ Length $(/ \mathrm{m})$

M Molar mass $\left(/ \mathrm{kg} \mathrm{mol}^{-1}\right)$.

$N$ Number $(/ 1)$.

$P \quad$ Perimeter $(/ \mathrm{m})$. 


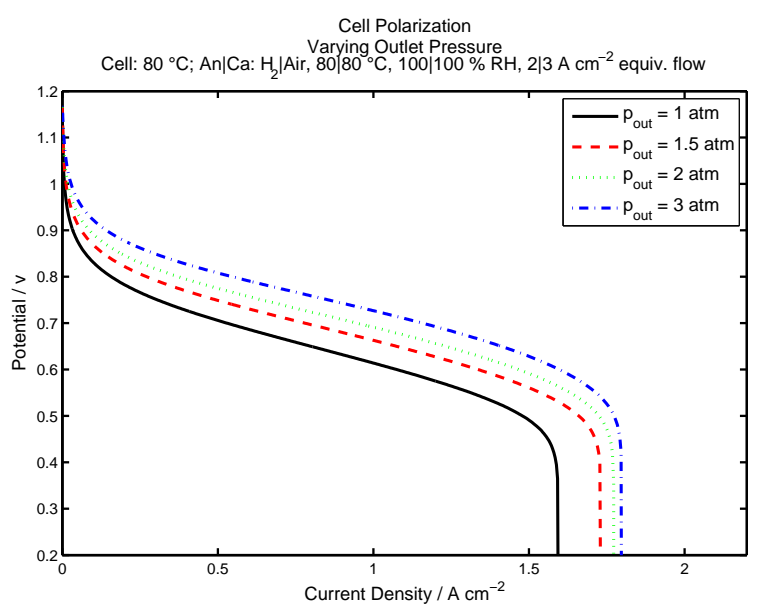

(a)

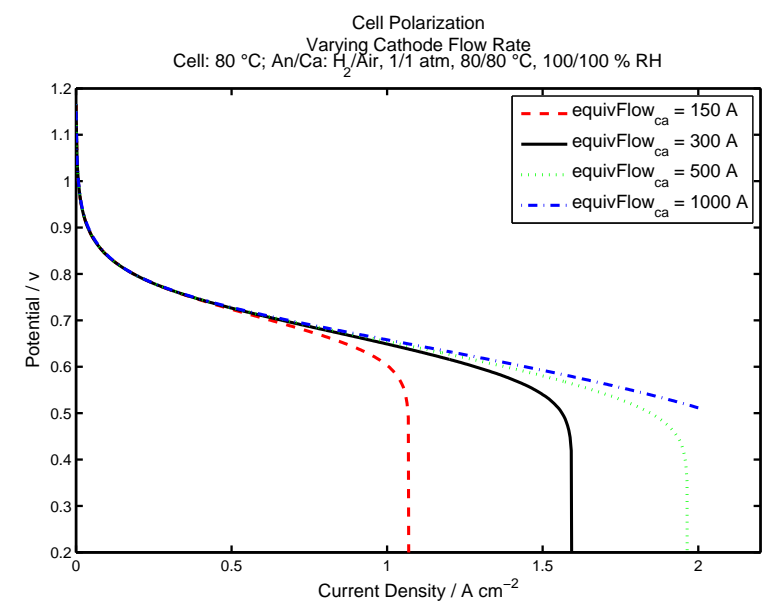

(c)

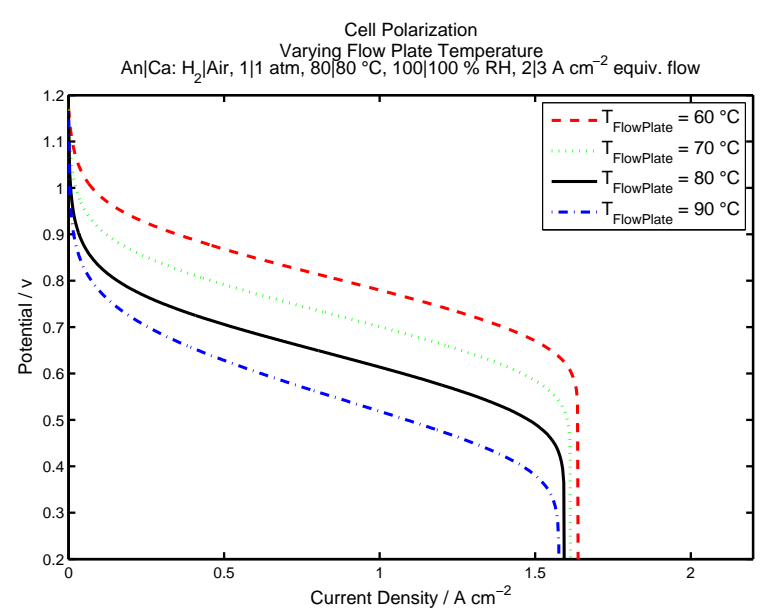

(b)

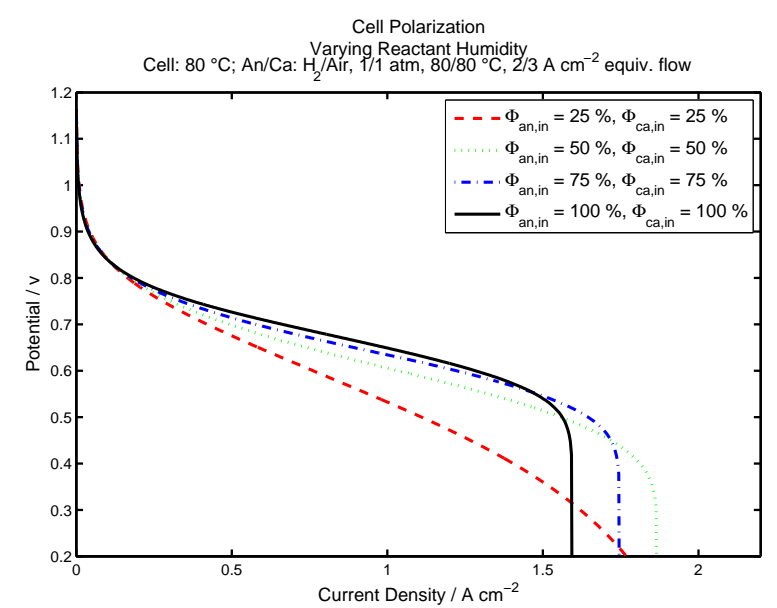

(d)

Figure 1: Polarization curves with varying (a) pressure at cathode and anode outlets, (b) temperature of anode and cathode flow plates, (c) reactant flow rate through the cathode flow channels, and (d) relative humidity at anode and cathode inlets.

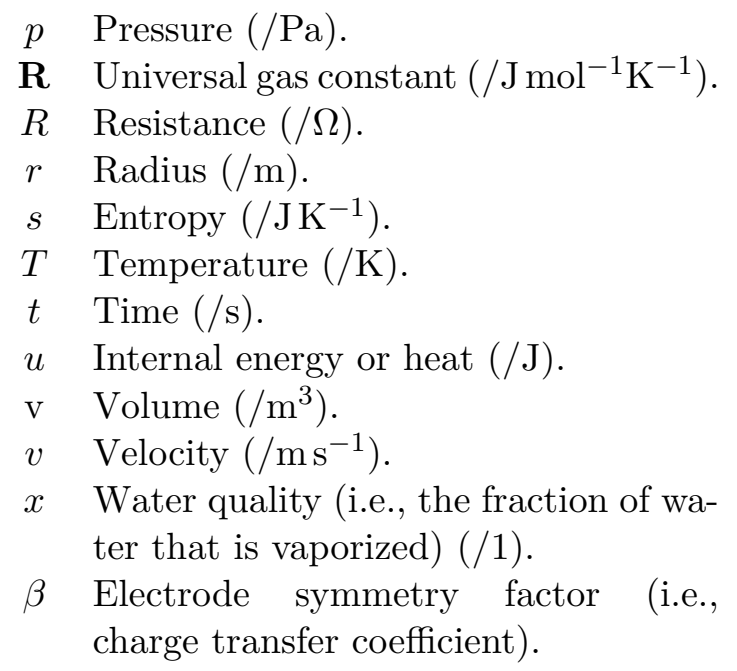

$\varepsilon \quad$ Porosity (fraction of free space to total space) $(/ 1)$.

$\gamma \quad$ Surface area per volume $\left(/ \mathrm{m}^{-1}\right)$.

$\kappa$ Permeability $\left(/ \mathrm{m}^{2}\right)$.

$\lambda$ PEM hydration $\left(/ \mathrm{mol}_{\mathrm{H}_{2} \mathrm{O}}\left(\mathrm{mol}_{\mathrm{SO}_{3}^{-}}\right)^{-1}\right)$.

$\mu$ Dynamic viscosity (/Pas).

$\nu$ Kinematic viscosity $\left(/ \mathrm{m}^{2} \mathrm{~s}^{-1}\right)$.

$\phi \quad$ Electric potential, (/V).

$\rho$ Density $\left(/ \mathrm{kg} \mathrm{m}^{-3}\right)$.

\section{Accents}

( ) Per amount $\left(/ \mathrm{mol}^{-1}\right)$.

( ) Per time (e.g., flow rate or velocity) $\left(/ \mathrm{s}^{-1}\right)$. 


$$
\begin{array}{ll}
()^{\prime \prime} & \text { Per area }\left(/ \mathrm{m}^{-2}\right) . \\
()^{\prime \prime \prime} & \text { Per volume }\left(/ \mathrm{m}^{-3}\right)
\end{array}
$$

\section{Subscripts}

$\begin{array}{cl}()_{(x)} & \text { As } x . \\ ()_{\cdot x} & \text { Along } x . \\ ()_{\nabla x} & \text { Due to the gradient in } x . \\ ()_{x, y} & \text { Of } x \text { and } y . \\ ()_{x} & \text { Of } x . \\ A & \text { On an areal basis. } \\ a n & \text { The anode. } \\ c a & \text { The cathode. } \\ d r y & \text { Without or excluding } H_{2} O . \\ F & \text { Degrees of freedom. } \\ g & \text { The gas phase. } \\ i & \text { The interface or chemical species de- } \\ & \text { noted by } i . \\ j & \text { The interface or chemical species de- } \\ & \text { noted by } j . \\ k & \text { The interface or chemical species de- } \\ & \text { noted by } k . \\ l & \text { The liquid phase. } \\ P & \text { Phases. } \\ r e a c t & \text { The reaction. } \\ S & \text { Species. } \\ \mathrm{v} & \text { On a volumetric basis. } \\ z & \text { The dimension from the anode to the } \\ & \text { cathode (parallel to charge flow). } \\ \leftarrow & \text { In the backward direction. } \\ \rightarrow & \text { In the forward direction. }\end{array}$

\section{Superscripts}

$+\quad$ In the positive state.

- In the negative state.

0 In the initial, nominal, or reference state (e.g., standard pressure).

eff Effective.

mod Modified.

\section{Acknowledgements}

This research was supported in its initial stages by the Office of Naval Research under grant \#N00014-04-0682 to the Hawaii Natural Energy Institute and later by the Robert G. Shackelford Fellowship of the Georgia Tech Research Institute. The authors wish to acknowledge the support of the Modelica Association and the Modelica community by the open sharing of the Modelica library and the Modelica_Fluid library. Although it is not currently possible to offer the library described within this paper online as open-source code, the authors request that any interested readers contact them.

\section{References}

[1] D. M. Bernardi and M. W. Verbrugge. A mathematical model of the solid-polymerelectrolyte fuel cell. Journal of The Electrochemical Society, 139(9):2477-91, 1992.

[2] J. O. M. Bockris, A. K. N. Reddy, and M. Gamboa-Aldeco. Modern Electrochemistry 2A: Fundamentals of Electrodics. Kluwer Academic/Plenum Publishers, New York, 2nd edition, 2000.

[3] B. McBride, M. Zehe, and S. Gordon. NASA Glenn coefficients for calculating thermodynamic properties of individual species. NASA report TP-2002-211556, 2002.

[4] Modelica Association. Modelica: A unified object-oriented language for physical systems modeling, February 22005.

[5] Modelica Association. Modelica Fluid Library, August 2006. v1.0 Beta 1.

[6] Modelica Association. Modelica Standard Library, March 2006. v2.2.1.

[7] M. J. Moran and H. N. Shapiro. Fundamentals of Engineering Thermodynamics. John Wiley \& Sons, Inc., Hoboken, NJ, 5th edition, 2004.

[8] Private communication from T. E. Springer, LANL. Fortran code of Springer 1991 polymer electrolyte fuel cell model, 2007.

[9] T. E. Springer, M. S. Wilson, and S. Gottesfeld. Modeling and experimental diagnostics in polymer electrolyte fuel cells. Journal of The Electrochemical Society, 140(12):35133526, 1993.

[10] T. E. Springer, T. A. Zawodzinski, and S. Gottesfeld. Polymer electrolyte fuel cell model. Journal of The Electrochemical Society, 138(8):2334-2342, 1991.

[11] J. X. Wang, T. E. Springer, P. Liu, M. Shao, and R. R. Adzic. Hydrogen oxidation reaction 
on $\mathrm{Pt}$ in acidic media: Adsorption isotherm and activation free energies. Journal of Physical Chemistry C, 111(33):12425-12433, 2007.

[12] A. Z. Weber and J. Newman. Modeling transport in polymer-electrolyte fuel cells. Chemical Reviews, 104(10):4679 - 4726, 2004.

[13] A. Z. Weber and J. Newman. Transport in polymer-electrolyte membranes III: Model validation in a simple fuel-cell model. Journal of The Electrochemical Society, 151(2):326$339,2004$.

[14] A. Z. Weber and J. Newman. Modeling gasphase flow in porous media. International Communications in Heat and Mass Transfer, 32(7):855 - 860, 2005.

[15] Wikipedia. Darcy's law, November 2008.

[16] Wikipedia. Maxwell-Stefan-diffusion, November 2008.

[17] Wikipedia. Fick's law of diffusion, April 2009.

[18] Z. Zhan, J. Xiao, Y. Zhang, M. Pan, and R. Yuan. Gas diffusion through differently structured gas diffusion layers of PEM fuel cells. International Journal of Hydrogen Energy, 32(17):4443-4451, 2007. 